\title{
Relationship between grain boundary segregation and grain boundary diffusion in $\mathrm{Cu}-\mathrm{Ag}$ alloys
}

\author{
R. K. Koju ${ }^{1}$ and Y. Mishin ${ }^{1}$ \\ ${ }^{1}$ Department of Physics and Astronomy, MSN 3F3, \\ George Mason University, Fairfax, Virginia 22030, USA
}

(Dated: July 8, 2020)

While it is known that alloy components can segregate to grain boundaries (GBs), and that the atomic mobility in GBs greatly exceeds the atomic mobility in the lattice, little is known about the effect of GB segregation on GB diffusion. Atomistic computer simulations offer a means of gaining insights into the segregation-diffusion relationship by computing the GB diffusion coefficients of the alloy components as a function of their segregated amounts. In such simulations, thermodynamically equilibrium GB segregation is prepared by a semi-grand canonical Monte Carlo method, followed by calculation of the diffusion coefficients of all alloy components by molecular dynamics. As a demonstration, the proposed methodology is applied to a GB is the $\mathrm{Cu}-\mathrm{Ag}$ system. The GB diffusivities obtained exhibit non-trivial composition dependencies that can be explained by site blocking, site competition, and the onset of GB disordering due to the premelting effect.

Keywords: Atomistic modeling; alloys; grain boundary segregation; grain boundary diffusion. 


\section{INTRODUCTION}

Solute segregation to grain boundaries (GBs) can affect many mechanical, thermodynamic and kinetic properties of materials [1]. Once at GBs, the solute atoms can diffuse through the material much faster than they would by regular, lattice diffusion mechanisms [2]. The accelerated atomic transport along GBs, often referred to as "short-circuit" diffusion, can control the kinetics of processes such as creep deformation [3 5], phase precipitation [6, 7], as well as complex kinetic phenomena such as dynamic strain aging [8, 9].

It is well-established that the self-diffusion and solute diffusion coefficients in GBs can exceed the lattice diffusion coefficients by many orders of magnitude, especially at low homologous temperatures [2]. What remains poorly understood is how the amount of GB segregation can affect the rate of GB diffusion. For example, in a binary alloy A-B one must consider GB diffusion coefficients of both the solute component $\mathrm{B}\left(D_{B}\right)$ as well as the host component A $\left(D_{A}\right)$. Several questions arise. For example, as the amount of GB segregation of $\mathrm{B}$ increases, do the diffusion coefficients $D_{A}$ and $D_{B}$ both increase, both decrease, or can show opposing trends? Which physical factors control the effect of segregation on GB diffusion? Can the segregation-diffusion relation change with temperature and/or alloy composition? How does the frequently occurring disordering of the GB structure at high temperatures can affect the segregation-diffusion relation?

To our knowledge, these questions remain largely open. Answering them by experiment is not impossible in principle but is hampered by technical obstacles. One of them being that, to keep track of both diffusion coefficients $\left(D_{A}\right.$ and $\left.D_{B}\right)$ in the same GB, a co-diffusion experiment is required with concurrent monitoring of the segregated amounts. The experiment would then have to be repeated for a set of alloy compositions and/or temperatures. Such experiments are technically challenging and, to our knowledge, have not been performed so far. Another challenge is related to the fact that GB diffusion experiments are predominantly carried out at relatively high temperatures at which a significant fraction of the atoms diffusing along the GB leaks into the surrounding lattice regions [2]. Under such conditions, called the type-B kinetic regime, one can only extract from the experiment the triple product $s D_{A, B} \delta, s$ being the segregation factor and $\delta$ the GB width. Separate deter-

mination of the GB diffusion coefficients $D_{A, B}$ requires specially designed low-temperature experiments conducted in the so-called type-C regime. C-regime measurements are much more difficult and have only been performed for a small number of systems [2, 10 15]. Such systems do not include alloys with a varied chemical composition. Furthermore, only the solute diffusivity $D_{B}$ has been measured in the C-regime.

Given the experimental challenges mentioned above, a meaningful alternative approach is offered by atomistic computer simulations. It has recently been demonstrated that GB diffusion coefficients can be reliably computed in pure metals as well as dilute binary alloys (in the latter case, for solute diffusion only) [16 19]. This methodology can serve as a 
starting point from which to launch a systematic study of the effect of GB segregation on GB diffusion of both chemical components in binary, and in the future multicomponent, alloy systems.

The goal of this paper is to initiate work in the outlined direction by performing a series of simulations of GB segregation and GB diffusion in $\mathrm{Cu}$-rich $\mathrm{Cu}-\mathrm{Ag}$ solid solutions chosen here as a model system. The $\mathrm{Cu}-\mathrm{Ag}$ system has the advantage of exhibiting a limited solid solubility of the two elements and a strong GB segregation trend. Its choice also puts us on a familiar ground since much information has already been obtained for this system in previous work [20 23]. In particular, a reliable interatomic potential is available [20], and the phase diagram predicted by this potential has been accurately computed [20, 23]. GBs in $\mathrm{Cu}$ have been studied extensively [17-19, 21, 24 37]. One typical GB was chosen here as an example, with the intent of extending this work to a larger set of boundaries in the future. We perform a detailed study of Ag GB segregation in a wide temperaturecomposition domain of the $\mathrm{Cu}-\mathrm{Ag}$ system, followed by a similarly detailed study of $\mathrm{GB}$ diffusion of both $\mathrm{Ag}$ and $\mathrm{Cu}$ and its correlation with the segregation behavior.

\section{METHODOLOGY}

Atomic interactions in the $\mathrm{Cu}-\mathrm{Ag}$ system were modeled using an embedded atom potential 20] that accurately reproduces a large number of physical properties of both $\mathrm{Cu}$ and $\mathrm{Ag}$. The potential was fitted to first-principles energies of $\mathrm{Cu}-\mathrm{Ag}$ compounds and predicts the $\mathrm{Cu}-\mathrm{Ag}$ phase diagram in reasonable agreement with experiment. Molecular dynamics (MD) simulations were performed using the Large-scale Atomic/Molecular Massively Parallel Simulator (LAMMPS) 38. The Monte-Carlo (MC) simulations utilized the parallel MC code ParaGrandMC developed by V. Yamakov at NASA [39]41].

As a representative high-angle GB, we chose the symmetrical tilt $\Sigma 17(530)[001]$ GB with the misorientation angle of $61.93^{\circ}$. Here, $\Sigma$ is the reciprocal density of coincident sites, [001] is the tilt axis, and (530) is the GB plane. The boundary was created in a rectangular periodic simulation block whose edges were, respectively, parallel to the tilt axis ( $x$-direction), normal to the tilt axis ( $y$-direction), and normal to the GB plane ( $z$-direction). The block had the approximate dimensions of $10.54 \times 10.48 \times 21.13 \mathrm{~nm}^{3}$ and contained $1.97 \times 10^{5}$ atoms. The ground-state structure of the GB in pure $\mathrm{Cu}$ was obtained by the $\gamma$-surface method [24, 25, 42]. The structure consists of identical kite-shaped structural units arranged in a zig-zag array as shown in Fig. 1. The rows of structural units running parallel to the tilt axis can be interpreted as closely spaced edge dislocations forming the GB core. The same structure of this GB was previously obtained in $\mathrm{Cu}[17,28]$ and $\mathrm{Ni}$ [43]. The GB energy was found to be $856 \mathrm{~mJ} / \mathrm{m}^{2}$ in agreement with previous reports [17, 28].

A prescribed amount of $\mathrm{Ag}$ was introduced into $\mathrm{Cu}$ by semi-grand canonical $\mathrm{MC}$ simulations implemented at a chosen temperature $T$ and a fixed value of the chemical potential 
difference between $\mathrm{Ag}$ and $\mathrm{Cu}$. The trial moves of the $\mathrm{MC}$ process included random displacements of randomly selected atoms with a random re-assignment of their chemical species to either $\mathrm{Ag}$ or $\mathrm{Cu}$. The trial move additionally included random changes in the dimensions of the simulation block with rescaling of the atomic coordinates to achieve zero pressure conditions in all three directions. The trial move was accepted or rejected by the Metropolis algorithm. The simulation produced a thermodynamically equilibrium distribution of $\mathrm{Ag}$ atoms in the GB region and inside the grains for the targeted alloy composition. The simulations covered the temperature range between $600 \mathrm{~K}$ and $1100 \mathrm{~K}$, with the alloy compositions varying from pure $\mathrm{Cu}$ to the solidus line.

The amount of $\mathrm{Ag}$ segregation was quantified by the excess number of $\mathrm{Ag}$ atoms per unit GB area at a fixed total number of atoms:

$$
\left[N_{\mathrm{Ag}}\right]=N_{\mathrm{Ag}}-N \frac{N_{\mathrm{Ag}}^{\prime}}{N^{\prime}},
$$

where $N_{\mathrm{Ag}}$ and $N_{\mathrm{Ag}}^{\prime}$ are the numbers of $\mathrm{Ag}$ atoms per unit area in two regions with and without the GB, respectively, and $N$ and $N^{\prime}$ are the respective total numbers of $\mathrm{Cu}$ and $\mathrm{Ag}$ atoms. Both regions were large enough to include both the GB and the interiors of the grains.

The degree of structural disorder in the GB was measured by the layer-averaged structure factor $S(\mathbf{k})$. The simulation block was divided into $0.1 \mathrm{~nm}$ thin layers parallel to the GB plane and numbered by index $i$. The structure factor corresponding to layer $i$ is defined by

$$
S_{i}(\mathbf{k})=\frac{1}{N_{i}} \sqrt{\sum_{j=1}^{N_{i}} \cos ^{2}\left(\mathbf{k} \cdot \mathbf{r}_{j}\right)+\sum_{j=1}^{N_{i}} \sin ^{2}\left(\mathbf{k} \cdot \mathbf{r}_{j}\right)},
$$

where $\mathbf{k}=2 \pi[2 / a, 0,0]$ is the chosen reciprocal lattice vector, $\mathbf{r}_{j}$ is the position of atom $j$ within the layer $i, a$ is the cubic lattice parameter, and $N_{i}$ is the total number of atoms in the layer. The structure factor so defined equals one in the perfect lattice at $0 \mathrm{~K}$, has a value $S_{\infty}(\mathbf{k})<1$ in the lattice at finite temperatures, and turns to zero in the liquid phase. It is expected to exhibit a local minimum at the GB position due to the local disorder. The value of the structure factor relative to the lattice value, $\varphi\left(z_{i}\right)=S_{i}(\mathbf{k})-S_{\infty}(\mathbf{k})$, is defined as the order parameter at position $z_{i}=\lambda i$ in the GB region ( $\lambda$ being the layer thickness). Furthermore, the width $w$ of the order parameter minimum can be taken as the structural width of the GB. Specifically, $w$ was defined as twice the standard deviation of the Gaussian fitted to the order parameter profile $\varphi\left(z_{i}\right)$ across the GB. Knowing the GB width, the Ag concentration in the GB can be found by averaging the atomic fraction of $\mathrm{Ag}$ over the layer of width $w$ centered at the Gaussian peak. This concentration provides a complementary measure of the GB segregation in addition to $\left[N_{\mathrm{Ag}}\right]$.

GB diffusion coefficients were computed from MD simulations performed on GBs preequilibrated by MC simulations. First, the potential energy peak across the current GB 
position was constructed by averaging the potential energy over thin layers parallel to the boundary plane. The peak width was typically around $1 \mathrm{~nm}$ or larger. Mean-square atomic displacements, $\left\langle x^{2}\right\rangle$ and $\left\langle y^{2}\right\rangle$, parallel to the GB plane were computed as functions of time for both $\mathrm{Ag}$ and $\mathrm{Cu}$ atoms. The calculations only included atoms within a $1 \mathrm{~nm}$ thick window centered at the boundary position. The mean-square displacements were monitored over a period of time $\Delta t$ ranging from $24 \mathrm{~ns}$ to $60 \mathrm{~ns}$, depending on the alloy composition and temperature. The GB diffusion coefficients of $\mathrm{Ag}$ and $\mathrm{Cu}$ in both directions were obtained from the Einstein relations $D_{x}=\left\langle x^{2}\right\rangle / 2 \Delta t$ and $D_{y}=\left\langle y^{2}\right\rangle / 2 \Delta t$, respectively. Due to the structural anisotropy of the GB, the diffusion coefficients parallel $\left(D_{x}\right)$ and normal $\left(D_{y}\right)$ to the tilt axis are generally different. To account for slight variations in the GB position with time due to thermal fluctuations, the $1 \mathrm{~nm}$ layer in which the mean-square displacements were calculated was periodically re-centered to the current GB position identified with the potential energy peak.

\section{RESULTS}

\section{A. Grain boundary segregation}

Fig. 2 illustrates typical equilibrium segregation profiles in the $\mathrm{Cu}-2$ at.\% alloy at various temperatures. The profiles were obtained by averaging the atomic fraction of Ag over thin layers parallel to the GB and then averaging over multiple snapshots saved during the $\mathrm{MC}$ simulations. Note that the segregation peak grows higher with decreasing temperature and broadens with increasing composition. As will be discussed below, the width of the segregation zone drastically increases near the solidus line as the GB undergoes the premelting transformation.

Representative order parameter profiles $\varphi(z)$ are shown in Fig. 3. At a fixed temperature (1100 K in this case), the minimum becomes deeper as Ag concentration increases, indicating the accumulation of structural disorder in the GB core. As the alloy composition approaches the solidus line, the order parameter in the GB tends to zero $(\varphi(0) \rightarrow 0)$, while the GB width $w$ rapidly increases and eventually spreads across the entire simulation block (Fig. (4). This behavior is a clear manifestation of GB melting and a sign that the alloy composition has reached the solidus line at the given temperature.

Isotherms of GB segregation are plotted in Fig. 5 using two measures of segregation: the total segregated amount $\left[N_{\mathrm{Ag}}\right]$ (number of excess Ag atoms per unit area) and the chemical composition (at.\% Ag) within the GB core. Both segregation parameters increase, in a nonlinear manner, with increase in the alloy concentration and decrease in temperature. Larger $\left[N_{\mathrm{Ag}}\right]$ values result from both the increase in the GB concentration and the GB broadening effect (Fig. 5a). By contrast, the isotherms shown in Fig. 5 b capture the behavior of the GB composition alone. Note that, at temperatures above the eutectic temperature predicted 
by the interatomic potential $\left(T_{E}=935 \mathrm{~K}[20]\right)$, the GB composition reaches the liquidus composition on the computed phase diagram [20]. Thus, at temperatures above $T_{E}$, the GB transforms into a liquid layer of the liquidus composition when the grain composition approaches the solidus line. GB melting behavior in the $\mathrm{Cu}-\mathrm{Ag}$ system was also noted in previous simulation studies [23, 44].

Distribution of the segregated Ag atoms inside the GB was examined in detail using the OVITO visualization software [45. In dilute compositions, the GB remained highly ordered and the segregated $\mathrm{Ag}$ atoms substituted for the host $\mathrm{Cu}$ atoms at particular positions within the GB structural units (Fig. 6a). As the alloy concentration increased, the GB structure grew increasingly disordered (Fig. 6b) until the structural units could no longer be distinguished (Fig. 6c). We emphasize that this disordering effect was entirely caused by the $\mathrm{Ag}$ segregation. In pure $\mathrm{Cu}$, the $\mathrm{GB}$ structure remained well-ordered until high temperatures approaching the $\mathrm{Cu}$ melting point (1326 K [46]).

\section{B. Grain boundary diffusion}

The GB diffusion coefficients were computed at temperatures and alloy compositions lying within the $\mathrm{Cu}$-based solid solution domain on the $\mathrm{Cu}-\mathrm{Ag}$ phase diagram. For the chosen GB, the diffusion coefficients parallel $\left(D_{x}\right)$ and normal $\left(D_{y}\right)$ to the tilt axis were found to be nearly equal. Thus, only the average values $D=\left(D_{x}+D_{y}\right) / 2$ are reported below.

The GB diffusion coefficients obtained are summarized on the Arrhenius diagrams, $\log D$ versus 1/T, shown Fig. $7 \mathrm{a}$ (Cu diffusion) and Fig. 7b (Ag diffusion). The alloy compositions are limited to 2 at.\% Ag to avoid close proximity of the solidus line. While diffusion in highly premelted GBs representing liquid layers could also be measured, the results would not be relevant to the segregation-diffusion relationship pursued in this work.

The diffusion coefficients in Fig. 7 reasonably follow the Arrhenius relation

$$
D=D_{0} \exp \left(-\frac{E}{k T}\right)
$$

at all temperatures. The plots demonstrate that the diffusion coefficients of both components depend on the alloy composition. To display the composition dependence more clearly, we plot the diffusion coefficients as a function of at.\% Ag in Fig. 8a. Two trends are obvious:

- Ag atoms diffuse in the GB slower than the host $\mathrm{Cu}$ atoms at low concentrations but faster at higher concentrations. The crossover occurs at about 1 at.\%Ag.

- While the Ag diffusion coefficients increase with Ag concentration monotonically, the $\mathrm{Cu}$ diffusion coefficients display a non-monotonic composition dependence, with a local minimum occurring at about 1 at.\% Ag. 
Note that the diffusion coefficients are shown in Fig. $8 \mathrm{a}$ on the logarithmic scale, meaning that the trends described are quite significant. The following explanation of these trends can be proposed. At low temperatures, the Ag atoms tend to segregate to particular GB sites offering the largest segregation energy. Due to this energetic preference, the Ag atoms spend most of the time occupying such favorable sites. They are reluctant to jump to alternate sites (i.e., against the driving force) to participate in the diffusion process, which results in slower diffusion rates. As additional Ag atoms segregate to the GB, they are forced to occupy less favorable (higher energy) sites and are more likely to contribute to the diffusion flux. In other words, the trapping effect weakens and $\mathrm{Ag}$ diffusion accelerates as the alloy concentration increases. At the same time, the $\mathrm{Cu}$ atoms diffuse slower with the addition of Ag due to the site blocking effect: the less mobile Ag atoms disrupt the fast diffusion pathways for $\mathrm{Cu}$ diffusion within the GB structure. As a result, the $\mathrm{Ag}$ and $\mathrm{Cu}$ diffusivities display opposite trends, converging toward each other as clearly observed in Fig. 8a.

This explanation only applies as long as the GB maintains an ordered structure with well-defined structural units offering distinct types of segregation site. This is certainly true for dilute alloy compositions as illustrated in Fig. 6a. At higher Ag concentrations when the GB develops a significant disorder (Fig. 6b) and eventually transforms into a liquid-like state (Fig. 6c), the situation changes. Diffusion in disordered GBs is governed by different atomic mechanisms from those in ordered structures [17, 47], hence a change in the diffusion trend with composition can be expected. This change can explain the crossover of the $\mathrm{Ag}$ and $\mathrm{Cu}$ diffusivities and the existence of a local minimum of the $\mathrm{Cu}$ GB diffusivity at about 1 at.\% Ag. This is the approximate composition at which the GB disordering commences at the temperatures studied here (Fig. 6b).

The crossover effect also manifests itself in the composition dependence of the activation energy $E$ of GB diffusion appearing in Eq.(3). While Ag GB diffusion is characterized by a higher activation energy in comparison with $\mathrm{Cu}$ below about 1 at.\% $\mathrm{Ag}$, the two activation energies converge to each other in more concentrated alloys in which the GB loses the ordered structure (Fig. 8b).

For validation of our methodology, we can compare the activation energies computed in this work with experimental data available in the literature (Table I). For GB self-diffusion in $\mathrm{Cu}$, only data for polycrystals is available [48]. The reported activation energy varies between $E=0.751 \mathrm{eV}$ and $0.878 \mathrm{eV}$, depending on the chemical purity of the material [48]. Our calculations predict $E=0.828 \mathrm{eV}$, which we consider a good agreement given that the polycrystalline value of $E$ represents an average over many GB types. For Ag GB diffusion, the experiments give $E=1.126 \mathrm{eV}$ (in pure $\mathrm{Cu}$ [14]) and $1.128 \mathrm{eV}$ (in $\mathrm{Cu}-0.2 \mathrm{at} . \% \mathrm{Ag}$ [15]), in both cases for polycrystalline samples. The closest chemical compositions studied in this work are $\mathrm{Cu}-0.12$ at.\% Ag and $\mathrm{Cu}-0.25$ at.\% Ag. The respective activation energies, $0.918 \mathrm{eV}$ and $0.967 \mathrm{eV}$, compare well with the experiment considering that they were obtained for 
one particular GB. Another piece of experimental information comes from a recent study of Ag diffusion in a Cu bicrystal with the $\Sigma 5(310)$ [001] GB [15]. Even though this boundary is different from ours and is considered special, the experimental activation energy $(0.983 \mathrm{eV}$ or $1.067 \mathrm{eV}$, depending on the diffusion direction) is close to our results for the $\Sigma 17(530)$ [001] boundary in the dilute limit. Thus, the comparison with experiment is very encouraging and lends confidence to the simulation results reported in this paper.

\section{CONCLUSIONS}

The goal of this work was to demonstrate that it is now possible to probe the effect of GB segregation on GB diffusion of both the solute and solvent components in alloys by means of atomistic computer simulations. The methodology proposed combines MC simulations to create an equilibration GB segregation with MD simulations to extract the GB diffusion coefficients. A reliable interatomic potential is required, and the relevant part of the phase diagram must be known or computed.

As an example, we have studied diffusion in a representative $\mathrm{GB}$ in the $\mathrm{Cu}-\mathrm{Ag}$ system in the temperature-composition domain of $\mathrm{Cu}$-based solid solutions. Our results indicate that the $\mathrm{GB}$ diffusivities of the solute $(\mathrm{Ag})$ and solvent $(\mathrm{Cu})$ atoms can exhibit quite different and non-trivial composition/temperature dependencies. They can correlate with each other, anti-correlate, cross, or have local minima. These behaviors reflect intricate interplays between different diffusion mechanisms and physical effects, such as site blocking and site competition. One factor that is more crucial in alloys than it is in elemental solids is the disordering of the GB structure. When the alloy composition and/or temperature approach the solidus line on the phase diagram, GBs can become atomically disordered at relatively low temperatures, eventually transforming to a liquid film [17, 23, 44, 49]. This disordering is fueled by GB segregation and can drastically alter the GB diffusion mechanisms and thus the segregation-diffusion relationship in comparison with ordered GB structures prevailing in elemental solids and/or solid dilute solutions.

This work was performed on one particular GB in one binary system. Future studies in the proposed direction may include larger GB sets, multicomponent systems, and a more detailed analysis of the underlying diffusion mechanisms.

Acknowledgement: This work was supported by the National Science Foundation, Division of Materials Research, under Award No. 1708314.

[1] A. P. Sutton, R. W. Balluffi, Interfaces in Crystalline Materials, Clarendon Press, Oxford, 1995. 
[2] I. Kaur, Y. Mishin, W. Gust, Fundamentals of Grain and Interphase Boundary Diffusion, Wiley, Chichester, West Sussex, 1995.

[3] R. L. Coble, A model for boundary diffusion controlled creep in polycrystalline materials, J. Appl. Phys. 34 (1963) 1679-1682.

[4] F. A. Mohamed, Y. Li, Creep and superplasticity in nanocrystalline materials: current understanding and future prospects, Mater. Sci. Eng. A 298 (2001) 1-15.

[5] Y. Mishin, J. A. Warren, R. F. Sekerka, W. J. Boettinger, Irreversible thermodynamics of creep in crystalline solids, Phys. Rev. B 88 (2013) 184303.

[6] J. W. Christian, The theory of transformations in metals and alloys, in: The Theory of Transformations in Metals and Alloys, volume 1, Pergamon Press, Oxford, UK, 2002, pp. 200-208, 269-273.

[7] D. A. Porter, K. E. Easterling, Phase transformations in metals and alloys, second ed., Taylor and Francis, 2004.

[8] C. Schwink, A. Nortmann, The present experimental knowledge of dynamic strain ageing in binary f.c.c. solid solutions, Mater. Sci. Eng. A 234 (1997) 1-7.

[9] W. A. Curtin, D. L. Olmsted, L. G. Hector, A predictive mechanism for dynamic strain ageing of aluminum-magnesium alloys, Nature Mater. 5 (2006) 875-880.

[10] Y. Mishin, C. Herzig, J. Bernardini, W. Gust, Grain boundary diffusion: fundamentals to recent developments, Int. Mater. Reviews 42 (1997) 155.

[11] Y. Mishin, C. Herzig, Grain boundary diffusion: recent progress and future research, Mater. Sci. Eng. A 260 (1999) 55-71.

[12] C. Herzig, S. V. Divinski, Grain boundary diffusion in metals: Recent developments, Mater. Trans. 44 (2003) 14-27.

[13] S. Divinski, M. Lohman, C. Herzig, Grain boundary diffusion and segregation of Bi in Cu: Radiotracer measurements in b and c diffusion regimes, Acta Mater. 52 (2004) 3973-3982.

[14] S. V. Divinski, M. Lohmann, C. Herzig, Ag grain boundary diffusion and segregation in Cu: Measurements in the types B and C diffusion regimes, Acta Mater. 49 (2001) 249-261.

[15] S. V. Divinski, H. Edelhoff, S. Prokofjev, Diffusion and segregation of silver in copper $\Sigma 5$ (310) grain boundary, Phys. Rev. B 85 (2012) 144104.

[16] M. R. Sørensen, Y. Mishin, A. F. Voter, Diffusion mechanisms in Cu grain boundaries, Phys. Rev. B 62 (2000) 3658-3673.

[17] A. Suzuki, Y. Mishin, Atomic mechanisms of grain boundary diffusion: Low versus high temperatures, J. Mater. Sci. 40 (2005) 3155-3161.

[18] T. Frolov, Y. Mishin, Molecular dynamics modeling of self-diffusion along triple junctions, Phys. Rev. B 79 (2009) 174110.

[19] T. Frolov, S. V. Divinski, M. Asta, Y. Mishin, Effect of interface phase transformations on diffusion and segregation in high-angle grain boundaries, Phys. Rev. Lett. 110 (2013) 255502.

[20] P. L. Williams, Y. Mishin, J. C. Hamilton, An embedded-atom potential for the $\mathrm{Cu}-\mathrm{Ag}$ 
system, Modelling Simul. Mater. Sci. Eng. 14 (2006) 817-833.

[21] T. Frolov, M.Asta, Y. Mishin, Segregation-induced phase transformations in grain boundaries, Phys. Rev. B 92 (2015) 020103(R).

[22] T. Frolov, M. Asta, Y. Mishin, Phase transformations at interfaces: Observations from atomistic modeling, Current Opinion in Solid State and Materials Science 20 (2016) 308315.

[23] J. Hickman, Y. Mishin, Disjoining potential and grain boundary premelting in binary alloys, Phys. Rev. B 93 (2016) 224108.

[24] A. Suzuki, Y. Mishin, Atomistic modeling of point defects and diffusion in copper grain boundaries, Interface Science 11 (2003) 131-148.

[25] A. Suzuki, Y. Mishin, Interaction of point defects with grain boundaries in fcc metals, Interface Science 11 (2003) 425-437.

[26] A. Suzuki, Y. Mishin, Atomic mechanisms of grain boundary motion, Mater. Sci. Forum 502 (2005) 157-162.

[27] J. W. Cahn, Y. Mishin, A. Suzuki, Duality of dislocation content of grain boundaries, Philos. Mag. 86 (2006) 3965-3980.

[28] J. W. Cahn, Y. Mishin, A. Suzuki, Coupling grain boundary motion to shear deformation, Acta Mater. 54 (2006) 4953-4975.

[29] Y. Mishin, A. Suzuki, B. Uberuaga, A. F. Voter, Stick-slip behavior of grain boundaries studied by accelerated molecular dynamics, Phys. Rev. B 75 (2007) 224101.

[30] D. E. Spearot, M. A. Tschopp, K. I. Jacob, D. L. McDowell, Tensile strength of $\langle 100\rangle$ and $\langle 110\rangle$ tilt bicrystal copper interfaces, Acta Mater. 55 (2007) 705-714.

[31] M. A. Tschopp, D. L. McDowell, Asymmetric tilt grain boundary structure and energy in copper and aluminium, Philos. Mag. 87 (2007) 3871 - 3892.

[32] M. A. Tschopp, D. L. McDowell, Structures and energies of $\Sigma 3$ asymmetric tilt grain boundaries in copper and aluminium, Philos. Mag. 87 (2007) $3147-3173$.

[33] M. A. Tschopp, G. J. Tucker, D. L. McDowell, Structure and free volume of $\langle 110\rangle$ symmetrical tilt grain boundaries with the E structural unit, Acta Mater. 55 (2007) 3959-3969.

[34] T. Frolov, Y. Mishin, Thermodynamics of coherent interfaces under mechanical stresses. II. application to atomistic simulation of grain boundaries, Phys. Rev. B 85 (2012) 224107.

[35] T. Frolov, D. L. Olmsted, M. Asta, Y. Mishin, Structural phase transformations in metallic grain boundaries, Nature Communications 4 (2013) 1899.

[36] S. J. Fensin, M. Asta, R. G. Hoagland, Temperature dependence of the structure and shear response of a $\Sigma 11$ asymmetric tilt grain boundary in copper from molecular-dynamics, Philos. Mag. 92 (2012) 4320-4333.

[37] T. Frolov, Y. Mishin, Phases, phase equilibria, and phase rules in low-dimensional systems, J. Chem. Phys. 143 (2015) 044706.

[38] S. Plimpton, Fast parallel algorithms for short-range molecular-dynamics, J. Comput. Phys. 
117 (1995) 1-19.

[39] V. Yamakov, The ParaGrandMC code can be obtained from the NASA Software Catalog: https://software.nasa.gov/software/LAR-18773-1. URL: https://software.nasa.gov/ software/LAR-18773-1.

[40] G. P. Purja Pun, V. Yamakov, Y. Mishin, Interatomic potential for the ternary Ni-AlCo system and application to atomistic modeling of the $\mathrm{B} 2-\mathrm{L} 1_{0}$ martensitic transformation, Model. Simul. Mater. Sci. Eng. 23 (2015) 065006.

[41] V. Yamakov, J. D. Hochhalter, W. P. Leser, J. E. Warner, J. A. Newman, G. P. Purja Pun, $\mathrm{Y}$. Mishin, Multiscale modeling of sensory properties of $\mathrm{Co}-\mathrm{Ni}-\mathrm{Al}$ shape memory particles embedded in an Al metal matrix, J. Mater. Sci. 51 (2016) 1204-1216.

[42] Y. Mishin, D. Farkas, Atomistic simulation of [001] symmetrical tilt grain boundaries in NiAl, Philos. Mag. A 78 (1998) 29-56.

[43] H. Sun, C. V. Singh, Temperature dependence of grain boundary excess free volume, Scripta Mater. 178 (2020) 71-76.

[44] P. L. Williams, Y. Mishin, Thermodynamics of grain boundary premelting in alloys. II. Atomistic simulation, Acta Mater. 57 (2009) 3786-3794.

[45] A. Stukowski, Visualization and analysis of atomistic simulation data with OVITO - the open visualization tool, Model. Simul. Mater. Sci. Eng 18 (2010) 015012.

[46] Y. Mishin, M. J. Mehl, D. A. Papaconstantopoulos, A. F. Voter, J. D. Kress, Structural stability and lattice defects in copper: Ab initio, tight-binding and embedded-atom calculations, Phys. Rev. B 63 (2001) 224106.

[47] Y. Mishin, An atomistic view of grain boundary diffusion, Defect and Diffusion Forum 363 (2015) 1-11.

[48] T. Surholt, Chr. Herzig, Grain boundary self-diffusion in $\mathrm{Cu}$ polycrystals of different purity, Acta Mater. 45 (1997) 3817-3823.

[49] Y. Mishin, W. J. Boettinger, J. A. Warren, G. B. McFadden, Thermodynamics of grain boundary premelting in alloys. I. Phase field modeling, Acta Mater. 57 (2009) 3771-3785. 
Table I: Activation energies of GB diffusion obtained by the present simulations in comparison with experimental data from the literature [12, 14, 15, 48]. For Ag GB diffusion in $\mathrm{Cu}-\mathrm{Ag}$ alloys, two chemical compositions are included as closest to the experimental composition of $\mathrm{Cu}-0.2 \mathrm{at} . \% \mathrm{Ag}$ [12. ${ }^{a}$ parallel to the tilt axis, ${ }^{b}$ normal to the tilt axis.

\begin{tabular}{|c|c|c|c|c|}
\hline Diffusing atoms & Material & GB type & Activation energy $E(\mathrm{eV})$ & Reference \\
\hline \hline $\mathrm{Cu}$ & $99.999 \% \mathrm{Cu}$ & Polycrystal & 0.878 & {$[48]$} \\
\hline $\mathrm{Cu}$ & $99.9998 \% \mathrm{Cu}$ & Polycrystal & 0.751 & {$[48]$} \\
\hline $\mathrm{Cu}$ & $\mathrm{Cu}$ & $\Sigma 17(530)[001]$ & 0.828 & This work \\
\hline $\mathrm{Ag}$ & $\mathrm{Cu}$ & Polycrystal & 1.126 & {$[14]$} \\
\hline $\mathrm{Ag}$ & $\mathrm{Cu}-0.2 \mathrm{at} . \% \mathrm{Ag}$ & Polycrystal & 1.128 & {$[12]$} \\
\hline $\mathrm{Ag}$ & $\mathrm{Cu}$ & $\Sigma 5(310)[001]$ & $0.983^{a} ; 1.067^{b}$ & {$[15]$} \\
\hline $\mathrm{Ag}$ & $\mathrm{Cu}-0.12$ at. $\% \mathrm{Ag}$ & $\Sigma 17(530)[001]$ & 0.918 & This work \\
\hline $\mathrm{Ag}$ & $\mathrm{Cu}-0.25$ at. $\% \mathrm{Ag}$ & $\Sigma 17(530)[001]$ & 0.967 & This work \\
\hline
\end{tabular}




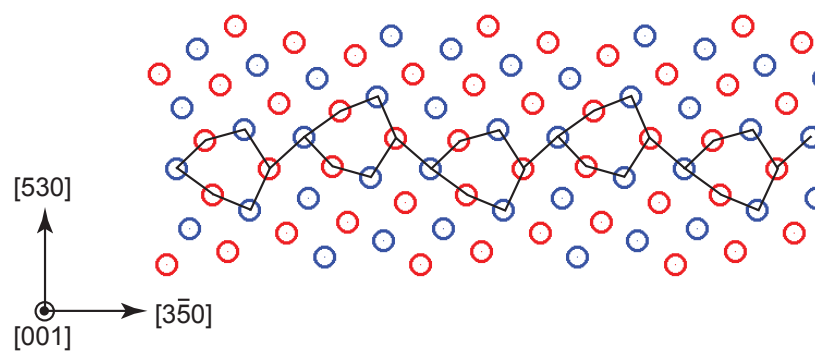

Figure 1: Atomic structure of the $\Sigma 17(530)[001]$ symmetrical tilt GB in $\mathrm{Cu}$. The red and blue circles represent the atoms in alternating (002) planes normal to the [001] tilt axis. The structural units are outlined.

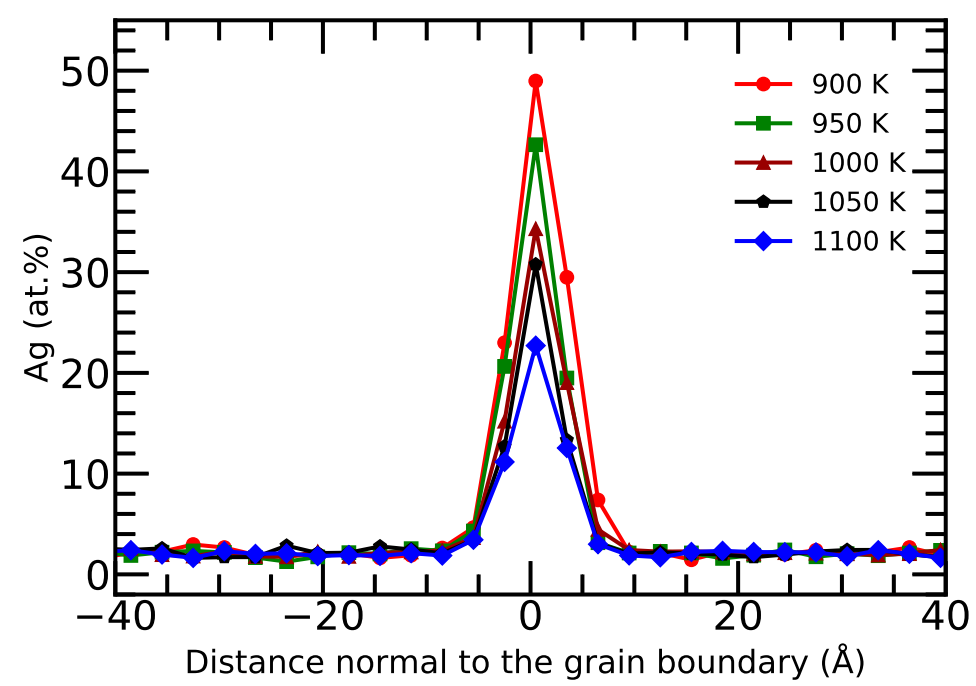

Figure 2: $\mathrm{Ag}$ GB segregation profiles in the $\mathrm{Cu}-2$ at.\% Ag alloy at various temperatures. 


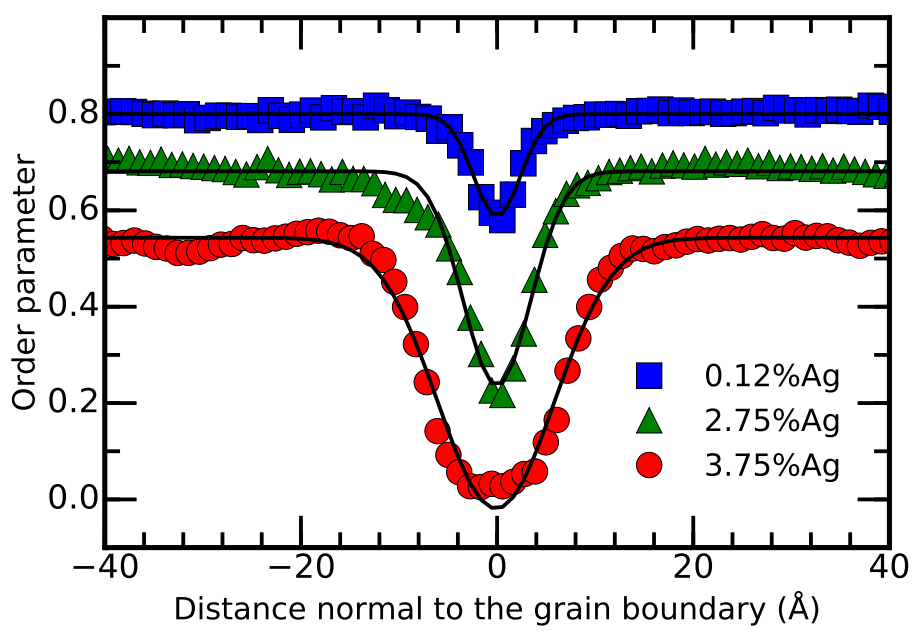

Figure 3: Representative profiles of the order parameter $\varphi(z)$ across the GB for three alloy compositions at the temperature of $1100 \mathrm{~K}$. The curves represent Gaussian fits of the local minimum occurring at the GB position. 


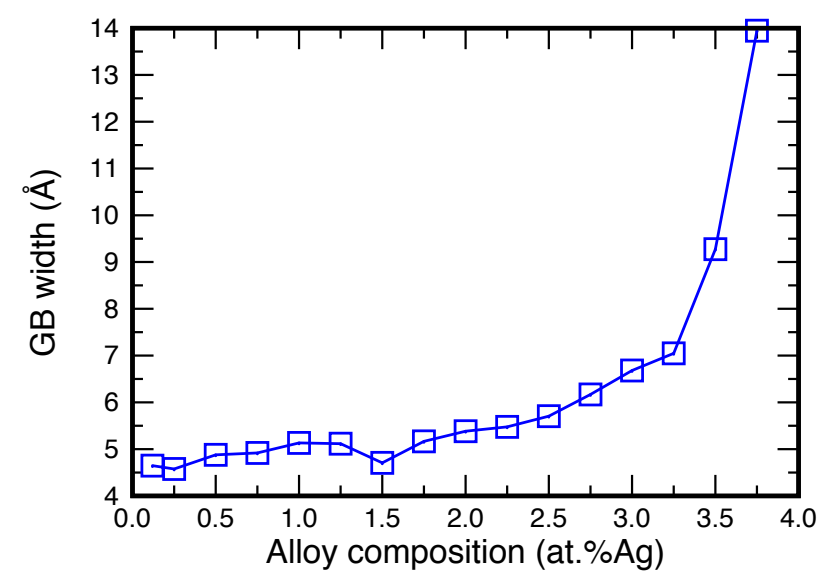

(a)

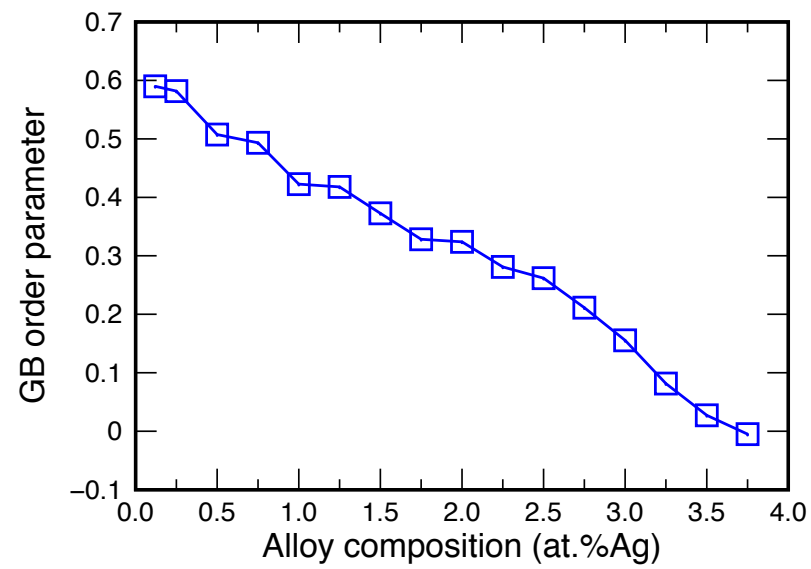

(b)

Figure 4: (a) GB width $w$ and (b) GB order parameter $\varphi(0)$ as functions of alloy composition at the temperature of $1100 \mathrm{~K}$. Note that $w$ diverges to infinity while $\varphi(0)$ tends to zero at the solidus composition of about 4 at.\% Ag. 


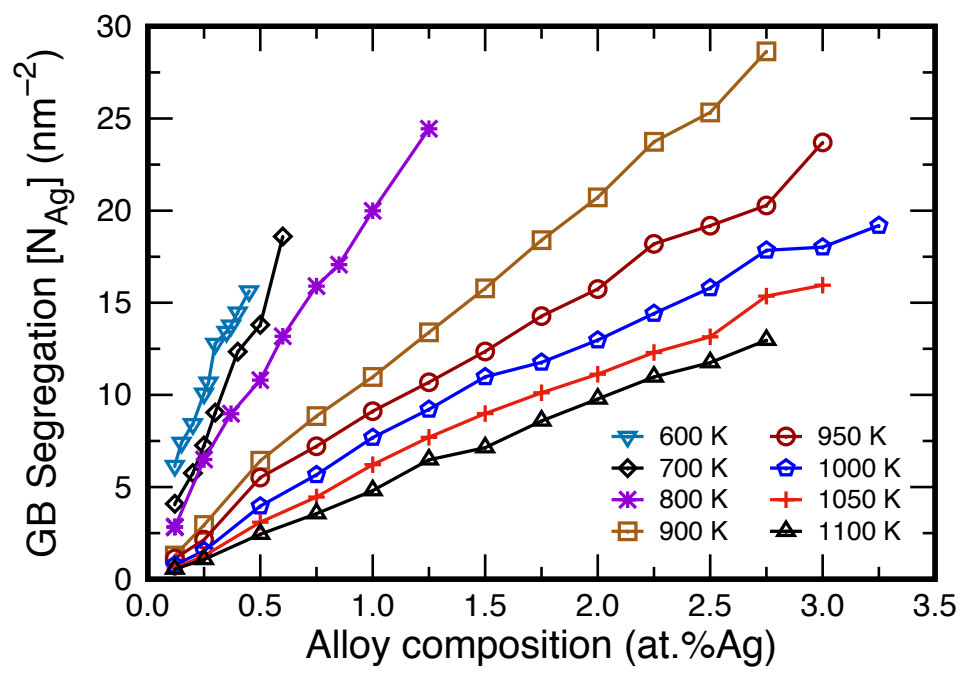

(a)

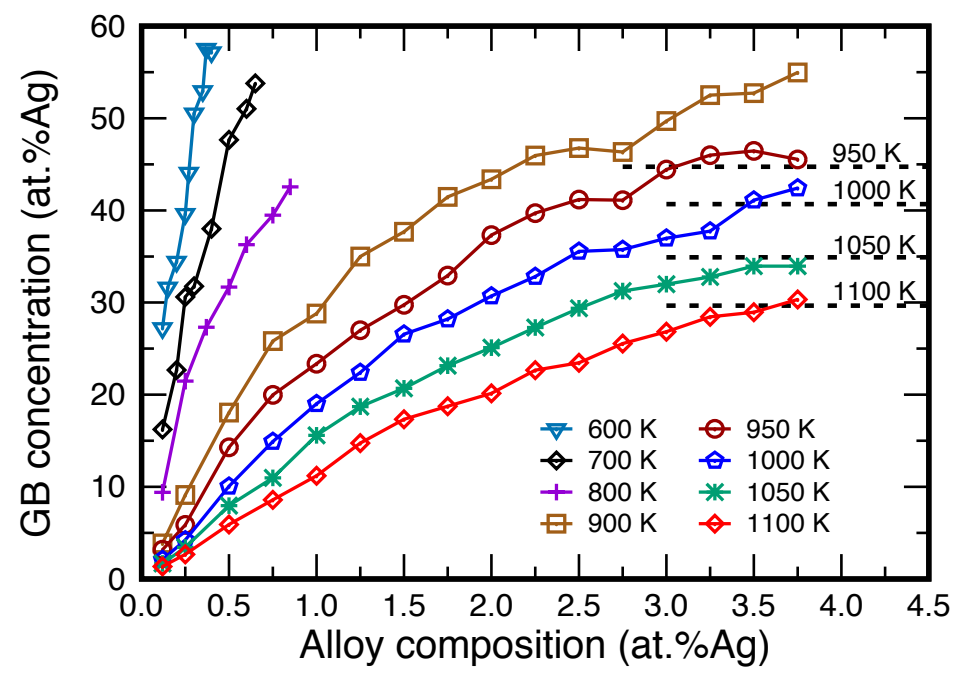

(b)

Figure 5: (a) Amount of Ag GB segregation $\left[N_{\mathrm{Ag}}\right]$ and (b) GB composition (atomic percentage of $\mathrm{Ag}$ atoms) as functions of alloy composition at different temperatures. Each curve ends at the solidus line on the phase diagram. In (b), the dashed lines represent the liquidus compositions obtained from the phase diagram at temperatures $\geq 950 \mathrm{~K}$. 

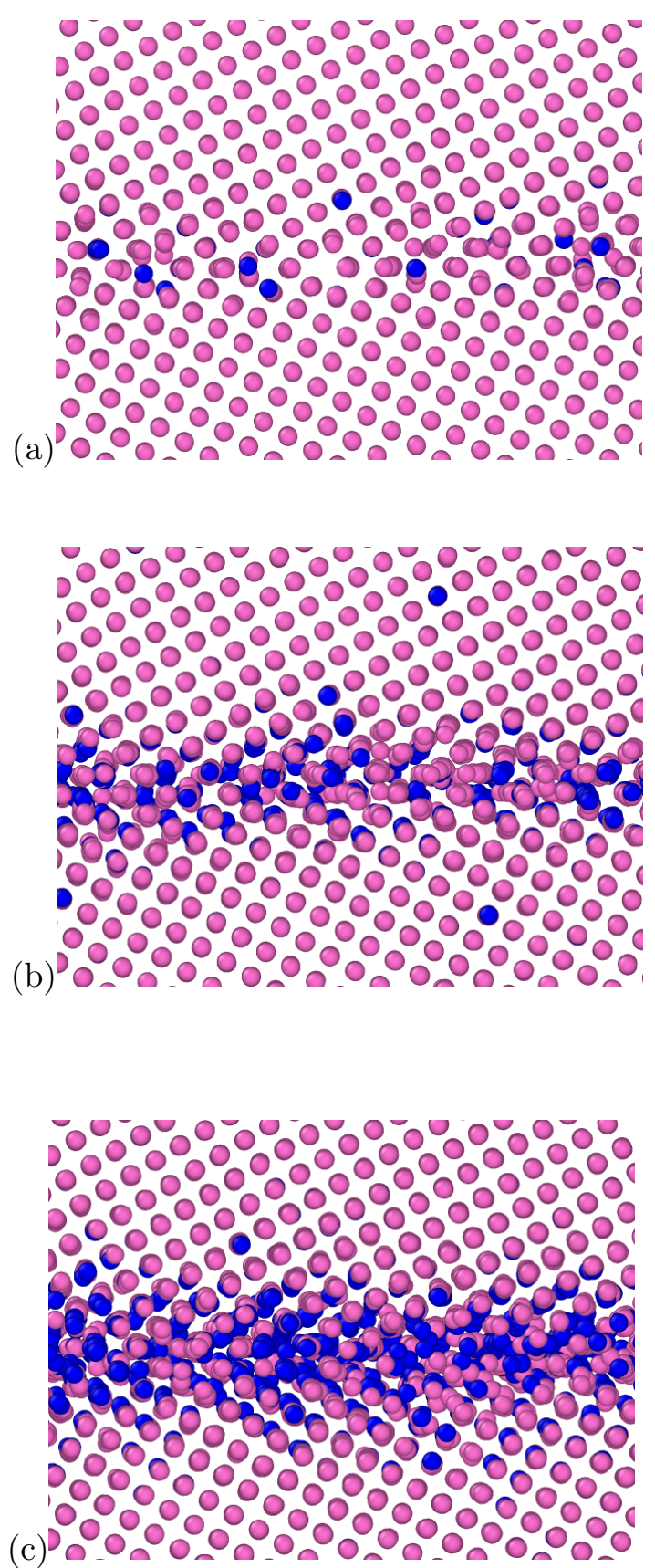

Figure 6: Distribution of Ag atoms in the GB at the alloy compositions of (1) Cu-0.12 at.\%, (b) $\mathrm{Cu}-1$ at.\% Ag, and (c) $\mathrm{Cu}-2$ at.\% Ag at the temperature of $900 \mathrm{~K}$. The $\mathrm{Ag}$ and $\mathrm{Cu}$ atoms are shown in blue and pink, respectively. Note the accumulation of GB disorder with increase in the GB segregation. 


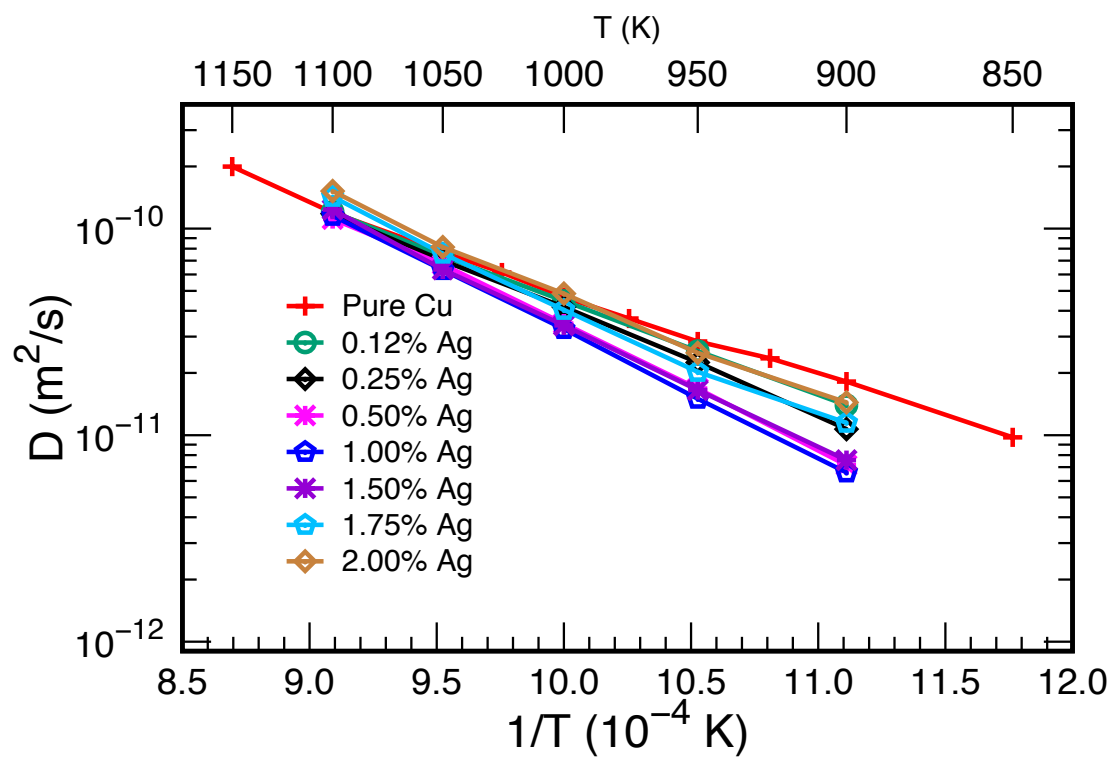

(a)

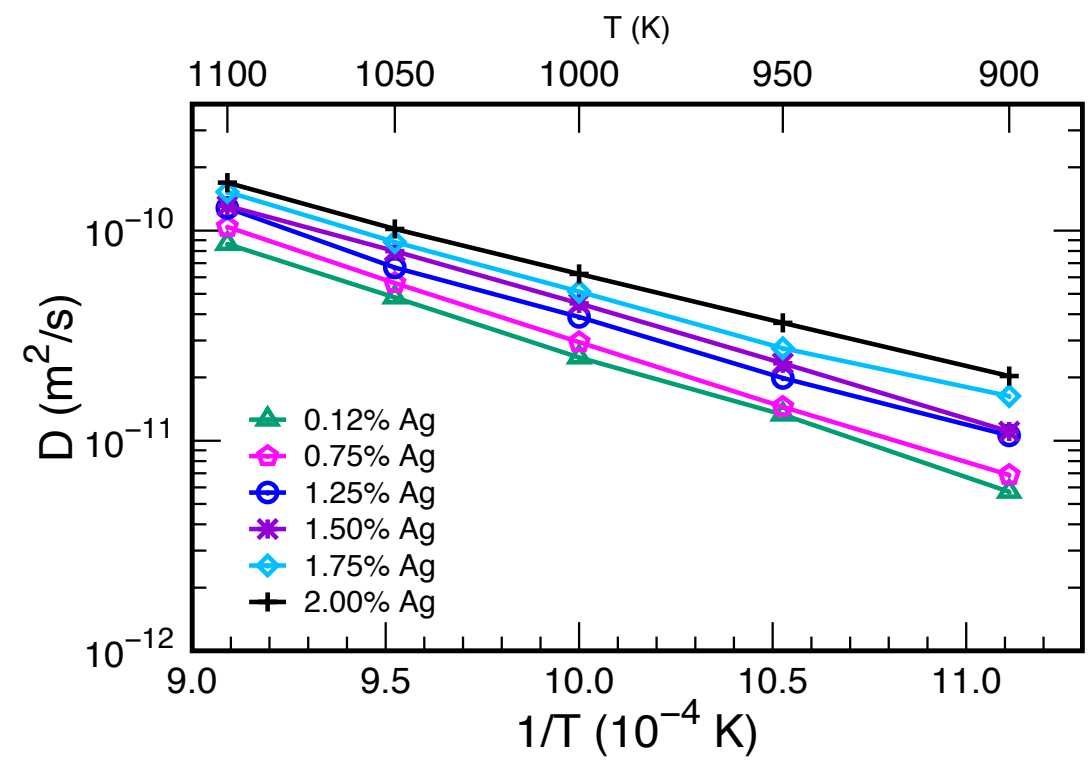

(b)

Figure 7: Arrhenius diagrams of GB diffusion coefficients of (a) $\mathrm{Cu}$ and (b) $\mathrm{Ag}$ in $\mathrm{Cu}-\mathrm{Ag}$ alloys with different chemical compositions. 


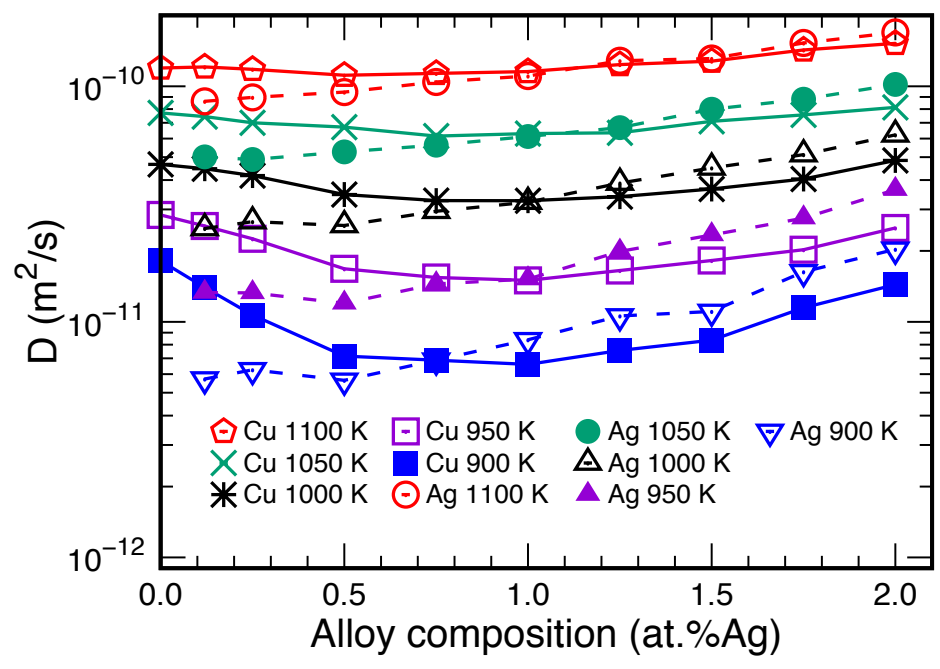

(a)

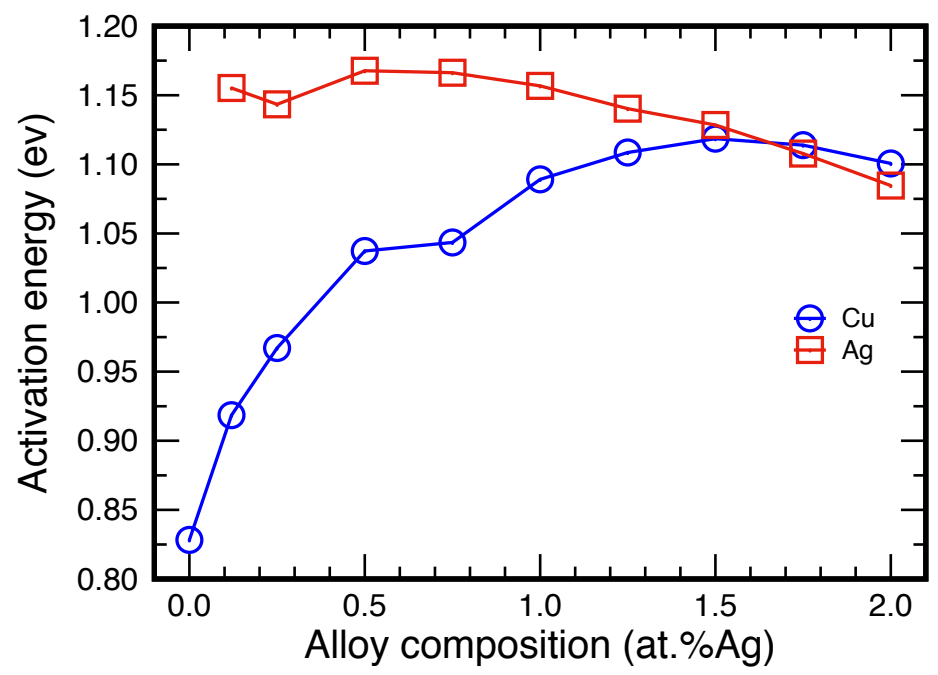

(b)

Figure 8: (a) GB diffusion coefficients of $\mathrm{Cu}$ and $\mathrm{Ag}$ as a function of alloy composition at different temperatures. The data points are connected by solid $(\mathrm{Cu})$ and dashed $(\mathrm{Ag})$ lines as a guide to the eye. (b) Activation energy of $\mathrm{Cu}$ and $\mathrm{Ag}$ GB diffusion as a function of alloy composition. 